\title{
Metástasis dural como manifestación inicial de cáncer de próstata
}

\section{Dural metastasis as the first manifestation of prostate cancer}

\author{
G. Alcalá-Cerra ${ }^{1}$, J.J. Gutiérrez-Paternina ${ }^{2}$, L.M. Niño-Hernández ${ }^{3}$, \\ R. Sabogal-Barrios ${ }^{1}$, L.R. Moscote-Salazar ${ }^{2}$, K. Suárez-Jaramillo ${ }^{4}$
}

\section{RESUMEN}

Las metástasis del cáncer de próstata en las meninges intracraneales son raras y con frecuencia son confundidas con meningiomas, hematomas epidurales o subdurales crónicos. Usualmente se presentan en pacientes con diagnóstico oncológico conocido en estadios avanzados de la enfermedad y solo en algunos raros casos sus manifestaciones han precedido a la detección del tumor primario. La presentación clínica es inespecífica, sin embargo, por la afinidad de estos tumores por la base del cráneo, constituye un diagnóstico diferencial del compromiso de pares craneales en varones mayores de 70 años.

El tratamiento de estas lesiones no ha sido estandarizado y dentro de las opciones terapéuticas están la resección quirúrgica, quimioterapia, radioterapia o la combinación de estas medidas y aún así la supervivencia es corta.

Se presenta el caso de un varón de 77 años cuya manifestación inicial del cáncer de próstata fue la sintomatología producida por una lesión metastásica en la duramadre, confirmada por histopatología. Se revisan los aspectos epidemiológicos, clínicos y de imagen más sobresalientes de las metástasis meníngeas del cáncer de próstata.

Palabras clave. Cáncer de próstata. Metástasis durales. Metástasis intracraneales. Hematoma subdural. Metástasis leptomeníngeas.

\begin{abstract}
Metastases of prostate cancer to intracranial meninges are rare and often confused with meningiomas or chronic subdural hematomas. These usually occur in patients with a known cancer diagnosis in advanced stages of the disease, and only in some rare cases do its manifestations precede the detection of the primary tumour. The clinical presentation is unspecific. However, due to the affinity of this tumour for the base of the skull, it must be included in the differential diagnosis of men over 70 years of age with cranial nerve palsy.

The treatment of these lesions has not been standardized. Within the therapeutic options we find surgical resection, chemotherapy, radiotherapy or a combination of these measures, and yet survival is poor.

We present the case of a 77 year old male patient whose initial symptoms of prostate cancer were caused by a metastatic lesion to the dura, confirmed by histopathology. We also review the epidemiological, clinical and imaging highlights of this condition.
\end{abstract}

Key words. Prostate carcinoma. Dural metastases. Intracranial metastases. Subdural hematoma. Leptomeningeal metastasis.
1. Departamento de Neurocirugía. Universidad de Cartagena. Colombia.

2. Facultad de Medicina. Universidad de Cartagena. Colombia.

3. Departamento de Patología. Universidad de Cartagena. Colombia.

4. Departamento de Medicina Interna. University of Texas Health Science Center. San Antonio. Texas.

Recepción: 18 de julio de 2011

Aceptación provisional: 24 de agosto de 2011

Aceptación definitiva: 5 de septiembre de 2011

\section{Correspondencia:}

Gabriel Alcalá Cerra

Subgerencia Quirúrgica

Hospital Universitario del Caribe

Barrio Zaragocilla, calle 29 \# 50-50

Cartagena de Indias. Colombia

E-mail: alcalagabriel@gmail.com 


\section{INTRODUCCIÓN}

Las lesiones metastásicas intracraneales del cáncer de próstata son raras. En diferentes estudios post-mortem se ha encontrado una prevalencia de 1 a $6 \%$, especialmente en pacientes portadores de tumores pobremente diferenciados y con enfermedad avanzada ${ }^{1,2}$.

Las metástasis en la duramadre pueden simular otras lesiones más frecuentes como meningiomas o hematomas subdura$\operatorname{les}^{2,3}$. Existen varios trabajos sobre casos, refiriendo pacientes que han sido sometidos a procedimientos quirúrgicos para drenaje de hematomas epidurales o subdurales crónicos; en los cuales los hallazgos sugestivos de una lesión tumoral solo fueron evidentes durante el acto quirúrgico ${ }^{2,4,5}$. La mayoría de casos corresponden a pacientes con diagnóstico oncológico conocido. En la revisión de la literatura comentada, sólo en tres casos las manifestaciones clínicas de las metástasis meníngeas precedieron al diagnóstico de cáncer de próstata ${ }^{1}$.

Se presenta el caso de un paciente cuyas manifestaciones iniciales del cáncer de próstata correspondieron a los síntomas producidos por una metástasis en la duramadre.

\section{CASO CLÍNICO}

Paciente masculino de 77 años que ingresa en el servicio de Urgencias por disartria, disfasia expresiva, hemiparesia derecha y deterioro progresivo del estado de conciencia. Fue informado por familiares de antecedentes de hipertensión arterial esencial en tratamiento con 50 miligramos de captopril cada 12 horas y aspirina 100 miligramos diarios. Adicionalmente, informaron que dos semanas antes a su ingreso presentó trauma occipital cerrado con pérdida transitoria del estado de conciencia, tras tropezar y caer; sin embargo, en aquel momento no acudió por atención médica.

A su ingreso presentaba intensa agitación motora, con tensión arterial 175/98 mm de $\mathrm{Hg}$, frecuencia cardiaca de 96 latidos por minuto, frecuencia respiratoria de 20 /minuto y temperatura de 36,4 grados centígrados. Se encontró una pequeña excoriación superficial en fase de cicatrización en la región parieto-occipital izquierda. Las pupilas eran iguales y reaccionaban simétricamente al reflejo foto-motor directo y consensual.
Al realizar estímulos dolorosos, realizaba apertura ocular, emitía palabras y localizaba el sitio estimulado, sin embargo, con hemiparesia derecha (Escala de coma de Glasgow: 10/15). El signo de Babinski estaba presente de forma bilateral.

Fue realizado un escáner cerebral de urgencia, previa administración de haloperidol para disminuir la agitación, la cual tuvo pobre calidad técnica dada la persistencia de los movimientos del paciente. En dicho estudio, fue observada una imagen extra-axial, hiperdensa en relación al parénquima cerebral, con forma semilunar de concavidad medial de 17 milímetros de espesor, localizada en la región frontal, parietal y occipital izquierda, la cual desviaba las estructuras de la línea media en 14 milímetros, la cual parecía corresponder con un hematoma subdural (Fig. 1). La magnitud desproporcionada del efecto de masa y el edema adyacente a la lesión alertaron sobre las posibles características neoplásicas de la lesión.

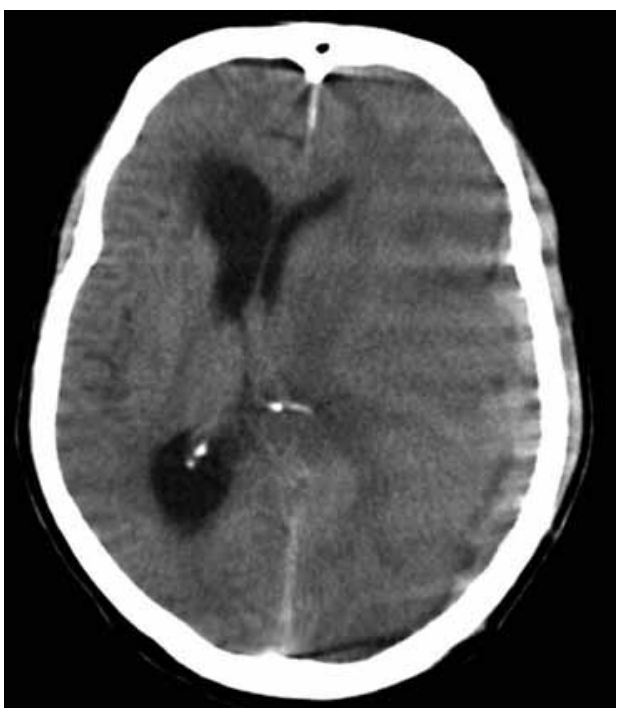

Figura 1. Tomografía axial computarizada cerebral simple que presenta imagen hiperdensa, semilunar hemisférica izquierda (se observan además artefactos por movimientos del paciente).

La tomografía cerebral con contraste demostró una imagen, extra-axial, hiperdensa en relación al parénquima cerebral, que ocupaba la superficie dorso-lateral fronto-temporo-parietal izquierda, con intensa captación del medio de contraste endovenoso, la cual infiltraba la profundidad de los surcos cerebrales frontales. Adicionalmente, en la ventana ósea se demostró adelgazamiento del cráneo en la región temporoparietal izquierda a causa de la lesión (Fig. 2). 


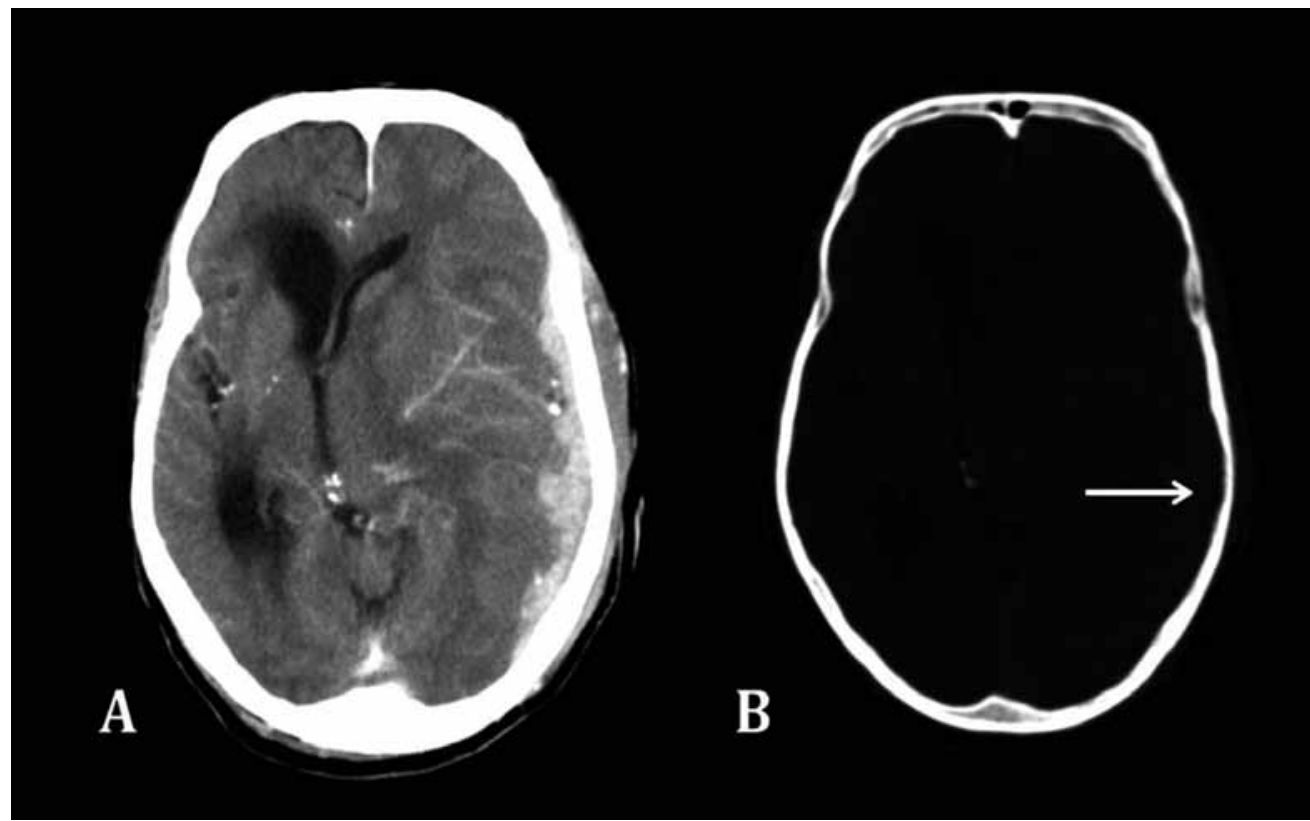

Figura. 2. A: Tomografía axial computarizada cerebral contrastada donde se observa la intensa captación del medio de contraste y la infiltración a los surcos cerebrales. B: La tabla interna del hueso temporal y parietal izquierdo estaba invadida (flecha).

Se realizó una craneotomía fronto-parietal izquierda durante la cual se encontró el hueso erosionado e infiltrado por una lesión violácea, firme, muy vascularizada, la cual se extendía a través de la duramadre, llegando a las leptomeninges, infiltrándolas y más profundamente al parénquima cerebral de la región frontal, temporal y parietal. Se realizó resección parcial, dejando material anormal adherido a la superficie cerebral.

El paciente presentó mejoría del estado de conciencia, alcanzando a estar alerta, con notable mejoría de la fuerza muscular en el lado derecho, aunque con persistencia de la disfasia sensitiva.

Ante la impresión clínica de una metástasis meníngea de un tumor primario desconocido fueron solicitadas tomografía con contraste de tórax, abdomen y pelvis, en los cuales solo se documento aumento del tamaño de la próstata. Al tacto rectal se encontró un nódulo prostático pétreo de bordes indefinidos a la palpación. El antígeno prostático específico sérico obtenido fue de $588 \mathrm{ng} / \mathrm{dL}$, por lo cual se realizó biopsia de próstata la cual demostró un adenocarcinoma pobremente diferenciado. El estudio histopatológico de la lesión intracraneal también correspondió a un adenocarcinoma metastásico pobremente diferenciado.

Por deseo de sus familiares, el paciente no fue sometido a ningún tratamiento oncológico complementario y fue dado de alta con cuidados ambulatorios paliativos.

\section{DISCUSIÓN}

Según los estudios basados en trabajos de autopsias realizadas entre los años 1950 y 1976, las metástasis en la duramadre intracraneal ocurren entre el 9 y 10\% de los pacientes con cualquier tipo de neoplasia maligna. Se estima que pueden ser el único sitio detectable de diseminación hasta en el $4 \%$ de los casos $^{4}$. El aumento en la incidencia de tumores malignos, aunado al incremento en la supervivencia de los pacientes con cáncer, se ha relacionado con una mayor frecuencia de metástasis durales; sin embargo, los estudios post-mortem más recientes las han detectado entre $8 \mathrm{a}$ $9 \%$ de los casos ${ }^{5}$. 
El cáncer de próstata usualmente se relaciona con metástasis óseas, distribuidas principalmente a lo largo de la columna vertebral y los huesos ilíacos. Debido a que las células neoplásicas se diseminan a través del plexo venoso epidural de Batson en sentido ascendente, las lesiones en la mayoría de los casos se ubican en las vértebras lumbares y sacras, mientras que la diseminación hacia estructuras intracraneales es rara $^{3,5}$. En la base de datos de pacientes con cáncer de próstata del Centro de Cáncer MD Anderson de los Estados Unidos, tras el análisis de 16.280 pacientes tratados en 54 años consecutivos, se encontró una incidencia de $0,63 \%^{6}$. Por su parte, las metástasis a las meninges cerebrales se ha encontrado con menor frecuencia. En una revisión sistemática de la literatura publicada hasta marzo de 2009 Guedes y col encontraron tan solo 35 casos, principalmente en pacientes con enfermedad metastásica avanzada y solo en 7 casos como la primera manifestación de la neoplasia ${ }^{1,7}$. Su presentación clínica es inespecífica y está en relación con las estructuras anatómicas ubicadas alrededor del tumor. La afección de pares craneales es más frecuente que en otros tumores metastásicos, ya que el cáncer de próstata tiende a invadir la base del cráneo donde sus fibras nerviosas encuentran sus agujeros de salida. Por ello, algunos autores han sugerido incluir al cáncer de próstata metastásico como uno de los diagnósticos diferenciales en varones mayores de 70 años con lesiones de pares craneales $^{1,3,8,9}$.

En la tomografía computarizada pueden encontrarse lesiones hiperdensas, nodulares o aplanadas dispuestas en el territorio de distribución de la duramadre. Existen varios casos simulando hematomas subdurales o epidurales en la tomografía cerebral simple, como en el presente caso. Incluso, ha sido descrita la presentación simultánea de la lesión tumoral con colecciones hemáticas extra-axiales, posiblemente ocasionadas por obstrucción del drenaje venoso dural o una respuesta angiodesmoplásica de la duramadre a la invasión por las células carcinomatosas ${ }^{3,5,10}$. Las imágenes de resonancia magnética son el método más sensible para su demostración, las cuales presentan un patrón típico ${ }^{1,4}$. En las secuencias T1 aparecen engrosamientos difusos de la duramadre que se refuerzan intensamente con gadolinio, asociados con múltiples lesiones grandes de aspecto nodular y edema cerebral perilesional ${ }^{1}$. También se observan metástasis en el cráneo adyacente $(70 \%)$, exhibiendo el signo de cola dural (44\%), edema vasogénico (53\%) y en el 34\% de los casos es posible demostrar invasión al parénquima cerebral ${ }^{4,11}$. Estas características explican los múltiples reportes de casos en los cuales han sido confundidas con meningiomas ${ }^{3,12-14}$.

El riesgo de diseminación meníngea está relacionado con el subtipo histológico. A pesar de que la mayoría de los informes de metástasis intracraneales corresponden a adenocarcinomas, los tumores de células pequeñas y cribiformes tienen una probabilidad 20 veces mayor que los adenocarcinomas de diseminación intracraneal ${ }^{6}$.

El tratamiento de estas lesiones no ha sido estandarizado. Dentro de las opciones terapéuticas están la resección quirúrgica, quimioterapia, radioterapia, terapias combinadas o solo cuidados de soporte. Con el arsenal terapéutico actual, tan solo se ha logrado una supervivencia media de 9,5 meses y un período libre de progresión de 3,5 meses $^{4}$.

Agradecimientos

A Carlos Valencia, del servicio de Radiología de la Clínica Universitaria San Juan de Dios de Cartagena, Colombia.

\section{BIBLIOGRAFÍA}

1. Guedes BD, Rocha AJ, Gama HP, Silva CJ. Dural metastases from prostate carcinoma: A systematic review of the literature a propos of six patients. Eur J Radiol 2011; 80: 236-240.

2. Tombin JM, Alleyne CH. Transdural metastasis from adenocarcinoma of the prostate mimicking subdural hematoma: case report. Surg Neurol 2002; 58: 329-331.

3. Cone LA, Koochek K, Henager HA, Fausel R, GADE-Andavolu R, PotTs BE et al. Leptomeningeal carcinomatosis in a patient with metastatic 
prostate cancer: case report and literature review. Surg Neurol 2006; 65: 372-375.

4. NAYAK L, ABREy LE, IWAMoto FM. Intracranial dural metastases. Cancer 2009; 115: 19471953.

5. Cheng YK, Wang TC, Yang JT, Lee MH, Su CH. Dural metastasis from prostatic adenocarcinoma mimicking chronic subdural hematoma. J Clin Neurosci 2009; 16: 1084-1086.

6. Tremont-Lukats IW, Bobustuc G, Lagos GK, Lolas K, Kyritsis AP, Puduvalli VK. Brain metastasis from prostate carcinoma the M.D. Anderson Cancer Center experience. Cancer 2003; 92: 363-368.

7. Sutton MA, Watkins HL, Green LK, Kadmon D. Intracranial metastases as the first manifestation of prostate cancer. Urology 1996; 48: 789-793.

8. Long MA, HusBAnd JE. Features of unusual metastases from prostate cancer. Br J Radiol 1999; 72: 933-941.

9. Izumi K, Mizokami A, Narimoto K, Sugimoto K, Koh E, Kumano T et al. Cranial nerve deficit caused by skull metastasis of prostate cancer: three Japanese castration-resistant prostate cancer cases. Int J Clin Oncol 2010; 15: 631-634.

10. Dorsi MJ, Zenonosa G, Hsua W, Huanga J. Dural prostate adenocarcinoma metastasis with subdural hematoma mimicking the appearance of an epidural hematoma. Clin Neurol Neurosurg 2010; 112: 501-504.

11. Confavreux CB, Cotton F, Tebib JG. Advanced MRI could help to differentiate meningeal carcinomatosis with mass effect from cerebral metastasis in prostate cancer. Bull Cancer 2006; 93: 31-35.

12. Tagle P, Villanueva P, Torrealba G, Huete I. Intracranial metastasis or meningioma? An uncommon clinical diagnostic dilemma. Surg Neurol 2002; 58: 241-245.

13. Lyons MK, Drazkowski JF, Wong WW, Fitch TR, NELSON KD. Metastatic prostate carcinoma mimicking meningioma: case report and review of the literature. Neurologist 2006; 12 : 48-52.

14. Lath CO, Khanna PC, Gadewar S, PatKar DP. Intracranial metastasis from prostatic adenocarcinoma simulating a meningioma. Australas Radiol 2005; 49: 497-500. 